\title{
EL NUEVO CANON A LAS UNIVERSIDADES: TRAS LA APROPIACIÓN DEL CANON DIGITAL PARA LAS COPIAS PRIVADAS, LA DEL OPEN ACCESS
}

\author{
Javier De-la-Cueva-González-Cotera
}



Javier De-la-Cueva-González-Cotera es licenciado en derecho y máster en estudios avanzados en filosofía por la Universidad Complutense de Madrid. Trabaja como abogado especializado en casos de propiedad intelectual y es profesor de la asignatura de propiedad intelectual en el Grado de creación musical en la Universidad Europea de Madrid. Se dedica además a programar proyectos tecnológicos y a impartir conferencias sobre GNU/Linux.

http://orcid.org/0000-0001-9152-2071

jdelacueva@icam.es

\section{Resumen}

La actual reforma de la Ley de propiedad intelectual incorpora la novedad de la creación de un canon a las universidades, que supone la apropiación de obras licenciadas bajo open access y su administración en el ámbito de los campus virtuales por la entidad de gestión Cedro. Se analiza un precedente normativo de la presente reforma legal, consistente en la regulación de la compensación por copia privada, que permitió a las entidades de gestión la apropiación durante años de dinero público. Dicho precedente tiene rasgos comunes con la actual reforma: el canon digital supuso la apropiación de lo público, mientras que el canon sobre las universidades representa la apropiación del procomún.

\section{Palabras clave}

Cedro, Entidades de gestión, Propiedad intelectual, Open access, OA, Conocimiento libre, Procomún.

Title: The new canon for universities: after the appropriation of the digital canon for private copies, now that of open access

\begin{abstract}
The current reform of Spain's copyright law incorporates a new levy on universities that is related to open access to publications. It will be collected by the Spanish agency Cedro (Centro Español de Derechos Reprográficos) and the virtual campuses of universities will be required to comply. We analyze a regulatory precedent in Spain for this legal reform, which levied a tax on potential private photocopying (for personal or "fair" use). This precedent has common features with the current reform: the levy on private copying was an appropriation of public access to print information, while the levy on universities implies the appropriation of the digital commons.
\end{abstract}

\section{Keywords}

Cedro, Collecting societies, Intellectual property, Open access, OA, Free culture, Commons.

De-la-Cueva-González-Cotera, Javier (2014). "El nuevo canon a las universidades: tras la apropiación del canon digital, la del open access". El profesional de la información, marzo-abril, v. 23, n. 2, pp. 183-189.

http://dx.doi.org/10.3145/epi.2014.mar.11

\section{Introducción}

Las entidades de gestión de la propiedad intelectual fueron diseñadas como unas organizaciones con fines loables: defender a los autores de las obras de propiedad intelectual frente a los abusos que se podrían encontrar en el uso de sus obras, así como recaudar los derechos en aquellos supuestos en los que el autor así voluntariamente lo disponía o en que era inviable que pudiera hacerlo por sí solo. Sin embargo, su función hoy en día se halla sometida a una profunda crítica, a la que no son ajenas situaciones como las que se documentan en este artículo. Las entidades de gestión, apartándose de sus iniciales funciones, sirven en demasiadas ocasiones como mera cadena de transmisión de los intereses de terceros ajenos a los autores y se integran en la tríada perversa señalada por Ferrajoli (2010, p. 271) de:

\footnotetext{
- dinero para hacer política e información;

- información para hacer dinero y política;

- política para hacer dinero e información.
} 
El proyecto de reforma del Texto refundido de la Ley de propiedad intelectual (Trlpi) aprobado por el Consejo de Ministros el 14 de febrero de 2014 es un ejemplo perfecto de lo señalado por Ferrajoli pues contiene diseños legales que suponen pagos, en especial a una de estas entidades, el Centro Español de Derechos Reprográficos (Cedro), organización comandada por los grandes grupos editoriales que a su vez son los titulares de los medios de comunicación. La reforma legal diseña en su favor dos pagos:

- uno, que no analizaremos, consistente en una tasa sobre los agregadores de noticias (cuyo objetivo principal es gravar el servicio de Google News);

- otro pago generado mediante la creación de un nuevo canon a las universidades por el uso de obras en el campus virtual.

Las entidades de gestión, apartándose de sus funciones iniciales, sirven en demasiadas ocasiones como cadena de transmisión de intereses de terceros ajenos a los autores

A lo largo de este artículo explicaremos el canon sobre las universidades pero, para comprender cómo se diseñan mecanismos apropiatorios, previamente analizaremos el caso de lo que mediáticamente se conoció como el "canon digital". Su síntesis es que durante años tuvo vigencia en nuestro país una norma por la que se gravaba la actividad de almacenamiento y procesado de información de forma indiscriminada. La norma finalmente fue declarada contraria al derecho europeo por lo que podría reclamarse la devolución de los pagos realizados hasta entonces. Sin embargo, para evitar estas reclamaciones por pagos indebidos, el Estado dictó el Real decreto 1657/2012 de 7 de diciembre, señalando que no procedía "la devolución del importe abonado o repercutido [...] satisfecho antes del 1 de enero de 2012", convalidando de esta manera todos los pagos hechos durante años en concepto de canon.

Tras este primer supuesto de apropiación de lo público, analizaremos cómo en la reforma del Trlpi se diseña una apropiación del procomún, lo que es de todos y no es de nadie (Gutiérrez, 2012). Para ejecutar esta segunda apropiación, el proyecto de reforma del Trlpi estipula la expropiación al autor de su derecho a decidir si entrega su obra a movimientos como el open access ${ }^{1}$, obligándose a que la gestión de estas obras en los campus virtuales se realice en exclusiva por una entidad de gestión, quien dispone de potestad unilateral para fijar las tarifas. Si bien quedan los trámites parlamentarios hasta que el proyecto sea ley, cualquiera que fuera el resultado final la voluntad del ejecutivo ya ha quedado reflejada.

\section{Apropiándose de lo público: el canon digital}

El 21 de octubre de 2010 el Tribunal de Justicia de la Unión Europea (TJUE, 2010) dictó la sentencia del caso Padawan contra la Sociedad General de Autores y Editores (Sgae). El TJUE resolvía lo que mediáticamente se conoció como el



Entre 1996 y 2011 los españoles tuvimos que abonar el injusto "canon digital" consistente en un sobreprecio que se aplicaba a todos los equipos, aparatos y materiales que se vendían en España susceptibles de usarse para hacer copias de obras intelectuales

"canon digital" (Aeval, 2008; CNC, 2009), consistente en un sobreprecio que se aplicaba a todos los equipos, aparatos y materiales que se vendían en España susceptibles de usarse para hacer copias de obras intelectuales. Los importadores y fabricantes de estos objetos satisfacían este sobreprecio a las entidades de gestión de la propiedad intelectual, quienes lo repartirían entre sus socios para así compensar a los autores, editores y productores por las ventas que se podían perder debido a las copias privadas realizadas por los usuarios de tales equipos, aparatos y materiales. Se trataba de un sobreprecio en cadena: los importadores y fabricantes se lo repercutían a los mayoristas, éstos a los minoristas y éstos al usuario final.

La sentencia del TJUE decretaba que el artículo 25 de nuestro Trlpi no era conforme con el artículo 5, apartado 2, letra b) de la Directiva 2001/29/CE del Parlamento Europeo y del Consejo, de 22 de mayo de 2001 (Unión Europea, 2001). A esta situación se llegó debido a que, cuando se promulgó el Trlpi (España, 1996), era una época en la que las tecnologías de registro utilizaban soportes variados, gravándose con canon aquellos cuyo uso estaba prácticamente predeterminado para copiar obras intelectuales. Sin embargo, cuando posteriormente se produjo una confluencia mayoritaria del sistema de registro en formato digital, la aplicación literal de nuestra regulación legal generó que la actividad cotidiana de almacenar información devengase un canon en favor de las entidades de gestión.

De esta manera se produjeron situaciones tan insólitas como que las grabaciones de los juicios orales en la jurisdicción civil, obligatorios en soporte videográfico conforme los artículos 147 y 187 de la Ley de enjuiciamiento civil, estuviesen tasadas con el canon. Las administraciones públicas, que son las que más soporte de información utilizan, fueron las grandes perjudicadas, hallándose sometida toda su actividad a un canon en favor de las entidades de gestión que, no olvidemos, se trata de asociaciones privadas. Las compras que las administraciones públicas realizaban de equipos, aparatos y materiales nunca estuvieron exentas de canon $^{2}$, que se repercutía por parte de los proveedores al Estado de la misma manera que lo repercutían a cualquier otro cliente. Esta situación se vio obligada a cambiar cuando 
el TJUE dictaminó (TJUE, 2010) que "la aplicación indiscriminada del canon por copia privada, en particular en relación con equipos, aparatos y soportes de reproducción digital que no se hayan puesto a disposición de usuarios privados y que estén manifiestamente reservados a usos distintos a la realización de copias privadas, no resulta conforme con la Directiva 2001/29". El TJUE establecía así que si los equipos, aparatos y soportes no se utilizaban para el ejercicio de la copia privada, no debían hallarse gravados. Como resultaba lógico, se eximía del canon a los objetos destinados a las actividades de empresas, administraciones públicas y profesionales.

La sentencia del caso Padawan afectó gravemente a las entidades de gestión de los derechos de propiedad intelectual de nuestro país, que vieron mermados sustancialmente sus ingresos. En palabras de Juan Mollá, presidente entonces de Cedro:

"A finales de año la junta directiva de la entidad decidió suspender temporalmente la mayoría de las actividades incluidas en la función social de Cedro. Esta decisión se debió a una medida de cautela por la incertidumbre que se plantea en la evolución de la recaudación de las cantidades de copia privada que sirven para financiar esta función de nuestra entidad, tras la sentencia del Tribunal Europeo de Justicia sobre copia privada (conocida como caso Padawan)" (Cedro, 2011, p. 9).

Los efectos de la sentencia no fueron inmediatos: todavía se tardó dos años en adaptar la legislación estatal a lo dispuesto por el TJUE. La modificación se llevó a cabo en dos etapas: en la primera produjo la derogación del canon (Real decreto 20/2011, de 30 de diciembre, disposición adicional décima), disponiéndose que el procedimiento de pago a los perceptores de la compensación equitativa por copia privada se establecería reglamentariamente por el Gobierno con cargo a los Presupuestos generales del Estado. Una vez suprimida la compensación por copia privada se procedió a regular el sistema de pago a cargo del Estado, mediante el Real decreto 1657/2012 de 7 de diciembre (España, 2012), que dispuso que la cuantía del canon se determinaría mediante orden del Ministro de Educación, Cultura y Deporte. En aplicación de esta disposición se dictó la Orden ECD/2128/2013, de 14 de noviembre (Espa$\tilde{n} a, 2013)$, en la que se dispuso que el importe del canon para el ejercicio 2012 sería de 8.636.728,09 euros.

La tardanza en adaptar nuestra legislación a la sentencia del caso Padawan (desde el 21 de octubre de 2010, fecha de la sentencia, hasta el Real decreto 20/2011, de 30 de diciembre), tuvo un efecto directo en los ingresos de las entidades de gestión ya que les permitió un año más obtener dinero por este concepto, lo que se aprecia claramente en la tabla 1, en la que observamos un cuadro de facturación por copia privada de las entidades de gestión en los ejercicios de 2009 a 2012.

No contentas con esta situación, las entidades de gestión interpusieron dos clases de acciones legales:

- denunciaron al Estado español ante la Comisión Europea en agosto de 2012 por contravenir según ellas el derecho europeo sobre copia privada (AIE, 2013, p. 94; Aisge, 2013, p. 9; Cedro, 2013, p. 32; Dama, 2013, p. 7; Egeda, 2013, p. 19; Vegap, 2013, pp. 13-15);

- presentaron dos demandas de responsabilidad patrimonial del Estado por los ejercicios 2011 y 2012 (Cedro, 2013, p. 32; Dama, 2013, p. 7; Egeda, 2013, p. 20; Vegap, 2013, pp. 14-15).

\section{El proyecto de reforma del Trlpi estipula la expropiación al autor de su derecho a decidir si entrega su obra a movimientos como el open access}

Se desconocen los resultados de estas acciones pero es relevante señalar, que si bien a las entidades de gestión no parece satisfacerles un sistema de pago directo a cargo de los presupuestos generales del Estado, nunca se quejaron del pago indirecto que suponía un sobreprecio a toda la actividad estatal, autonómica, local, empresarial y profesional.

Por su parte, el Estado blindó a las entidades de gestión: si la normativa estatal era contraria a la europea, ello implicaba que durante años se había estado pagando un importe contrario a la ley que podría ser solicitado por quienes lo hubieran satisfecho. Para evitar que esto sucediera, en la disposición transitoria primera del mencionado Real decreto $1657 / 2012$, de 7 de diciembre, se establecía que "se entenderá que no procede la devolución del importe abonado o repercutido por la aplicación del régimen de compensación equitativa por copia privada a los fabricantes, distribuidores, mayoristas o minoristas y compradores finales que lo

Tabla 1. Facturación por copia privada de las entidades de gestión

\begin{tabular}{|l|c|c|c|c|}
\hline \multicolumn{1}{|c|}{ Entidad } & $\mathbf{2 0 0 9}$ & $\mathbf{2 0 1 0}$ & $\mathbf{2 0 1 1}$ & \multicolumn{1}{c|}{$\mathbf{2 0 1 2}$} \\
\hline Cedro & 21.981 .801 & 19.923 .231 & 19.292 .339 & 4.891 .260 \\
\hline Dama & 190.874 & 252.880 & 219.033 & 28.356 \\
\hline Sgae & 27.677 .000 & 28.156 .000 & 20.247 .000 & 4.231 .000 \\
\hline Vegap & 3.412 .098 & 2.787 .572 & 2.833 .494 & 789.553 \\
\hline AIE & 8.978 .970 & 9.395 .535 & 7.611 .750 & 1.552 .470 \\
\hline Aisge & 11.898 .935 & 12.756 .031 & 8.436 .287 & 1.102 .282 \\
\hline Agedi & 6.498 .300 & 6.198 .400 & 5.295 .600 & 1.634 .700 \\
\hline Egeda & Sin datos & Sin datos & Sin datos & Sin datos \\
\hline Total & 80.637 .978 & 79.469 .649 & 63.935 .503 & 14.229 .621 \\
\hline
\end{tabular}

Fuente: Memorias e informes de las entidades de gestión (Cedro, 2010, p. 20-21; 2011, p. 21; 2012, p. 81; 2013, p. 66; Dama, 2011, p. 23; 2012, p. 20; 2013, p. 20; Sgae, 2010, p. 93; 2011, p. 97; 2012, p. 41; 2013, p. 41; Vegap, 2010, p. 7; 2011, p. 7; 2012, p. 7; 2013, p. 7; AIE, 2010, p. 94; 2011, p. 118; 2012, p. 124; 2013 , p. 97; Aisge, 2010, p. 25; 2011, p. 25; 2012, p. 26; 2013, p. 22; Agedi, 2010, p. 8; 2011, p. 8; 2012, p. 8; 2013, p. 8).

En el caso de Vegap, las cantidades presentadas se obtienen de la suma de las dos cantidades que figuran en los informes en concepto de copia privada: reprográfica y audiovisual. 




Se apropian del open access e impiden a los autores decidir sobre sus derechos

hubieran satisfecho antes del 1 de enero de 2012". Evitando la posibilidad de reclamación de lo indebidamente pagado se consumaba la apropiación de lo público bajo la apariencia del pago de derechos de autor y convalidando lo que en la práctica fue una póliza sobre el papel del siglo XXI, el soporte digital, pagadera por todos en favor de unas asociaciones privadas.

\section{Apropiándose del procomún: el open access}

Ante esta situación de crisis, Cedro apuntó nuevamente al Estado como fuente de ingresos. Ya en la Memoria del ejercicio 2010 señaló sus intenciones (Cedro, 2011, p. 29):

“Desde hace varios años, Cedro está tratando de que la Administración y el sector educativo en todos sus niveles, enseñanza reglada preuniversitaria y universitaria, remuneren a los autores y editores por la reutilización de los contenidos de sus obras a través de las licencias de nuestra entidad.

Prácticamente ningún ministerio ni organismo público de carácter autonómico o local cuenta con licencia de Cedro. Este hecho se ha puesto en conocimiento de forma sistemática a las autoridades competentes sin que se haya obtenido respuesta definitiva por su parte. En 2009 se envió al Ministerio de Presidencia una propuesta de regularización global que, a día de hoy, no ha sido contestada".

En igual sentido se expresaba la Memoria del año 2011 (Ce$d r o, 2011$, p. 35), sin que conste ya en la de 2012 referencia alguna a ministerios, organismos públicos de carácter autonómico o local, a la necesidad de estos órganos de obtener licencia concedida por Cedro, ni a respuesta alguna o silencio del Ministerio de la Presidencia. Sin embargo, sí constan las referencias a los tres procedimientos judiciales que Cedro interpuso contra las universidades Autònoma de Barcelona, de Barcelona y Carlos III de Madrid (Cedro, 2012, p. 33) y que supusieron la excusa para la siguiente etapa de apropiación: la "apropiación del procomún" mediante el canon sobre el open access.

- Cedro decía representar a unos 20.000 socios, mientras que las universidades en su conjunto cuentan con 130.000 docentes e investigadores;

- mantenía que representaba los derechos de unos 4 millones de obras, mientras que en WorldCat, el catálogo colectivo de $O C L C$, existen unos 300 millones de registros; no practicó ninguna prueba sobre la encomienda de gestión de los derechos de los autores o traductores en lo referente a copias y puesta a disposición digital de las obras literarias.

El centro de los litigios consistía en saber quién es el responsable de que determinados profesores pusieran a disposición de sus alumnos textos digitalizados alojándolos en el campus virtual pero también se discutía la representatividad de Cedro ya que su número de socios y número de obras en comparación con los existentes es mínimo. Y con respecto a los derechos digitales las circunstancias son aún peores para esta entidad.

En 2004, Cedro adaptó sus contratos para incluir la gestión de los derechos sobre copias digitales y su puesta a disposición online. A partir de esa fecha los nuevos socios les encargarían no sólo la gestión de los derechos sobre copias reprográficas sino también digitales. Sin embargo, con los derechos anteriores a 2004 existía un problema grave. Cedro gestiona derechos tanto de socios (por encargo directo de éstos) como de autores no socios (que han cedido sus derechos a las editoriales que a su vez se los han cedido a Cedro). Con respecto a sus socios, Cedro circularizó la petición de adhesión a sus nuevas modalidades de gestión pero con respecto a los no socios, nada podía hacer puesto que eran las editoriales quienes por su parte tenían que adaptar sus contratos con los autores para nuevas obras así como solicitar los derechos a los autores de obras antiguas. Las universidades conocían bien este problema puesto que mantienen editoriales y cuando se celebró la prueba en los procedimientos contra las universidades Autònoma de Barcelona y de Barcelona, si bien Cedro trajo al procedimiento documentos de gestión de derechos editoriales, no trajo ninguno de derechos autorales o de traductores.

\section{La norma estatal es contraria al derecho de la Unión Europea, pero las entidades de gestión seguirán cobrando hasta que una resolución del TJUE anule la legisla- ción del Estado}

Ante estas evidencias, el Ministerio de Educación, Cultura y Deporte acudió en auxilio de Cedro: la gestión que esta entidad nunca había obtenido contractualmente la iba a conseguir mediante la modificación del Trlpi, en cuyo anteproyecto se incorporó un artículo 32, apartado 3, que creaba un derecho irrenunciable en favor del autor de manuales universitarios o publicaciones asimiladas a cobrar una remuneración equitativa que se haría efectiva a través de las entidades de gestión. Sin perjuicio de la imprecisión de conceptos tales como publicaciones asimiladas, la reforma no dejaba dudas: Cedro se encargaría de la gestión de todos los derechos, teniendo facultades unilaterales para fijar las tarifas, actualmente señaladas en 5 euros por alumno, lo que supondría un gasto público anual de 5,92 millones de euros (Crue, 2013, p. 17). 
Se demostraba una vez más lo que Benkler señalaba (2006, p. 461), que las expansiones de los derechos operan, en la práctica, "como una tasa sobre modelos de producción no propietarios en favor de modelos propietarios. Hace que el acceso a los recursos de información sean más caros para todos, mientras que incrementa la apropiación solamente para algunos".

La Conferencia de Rectores de las Universidades Españolas (Crue) realizó el 17 de abril de 2013 sus alegaciones al texto del anteproyecto señalando (Crue, 2013, p. 13) su especial preocupación por la apropiación del open access y sus efectos de empobrecimiento por su incidencia en la ciencia y en la tecnología:

"Mediante el texto propuesto en el Anteproyecto, se impide al autor ejercitar uno de sus derechos esenciales como es el de disponer como quiera de sus derechos de explotación. La creación de un derecho irrenunciable, lejos de suponer una ventaja para el autor, supone un atentado a la posibilidad de que se incorpore a movimientos como el del acceso abierto [...]

La ciencia y la tecnología se fundamentan en la posibilidad de difusión y de refutación. Impedir a los autores la decisión sobre sus derechos, haciéndolos irrenunciables, obligando a su gestión a través de una entidad sin representatividad en el ámbito universitario y permitiendo a dicha entidad la fijación unilateral de los precios supondrá sin duda un empobrecimiento de la capacidad de difusión y de refutación de la ciencia y de la tecnología en nuestro país, con el empobrecimiento correspondiente".

El Consejo de Estado se pronunció sobre la reforma. En su Dictamen 1064/2013 de fecha 11 de noviembre, sobre el texto del anteproyecto de reforma del Trlpi, mantuvo una postura crítica con el texto del Ministerio de Educación, Cultura y Deporte. Según el Consejo de Estado (2013, 5.2), "conviene no olvidar que en el sector de la docencia e investigación convive el derecho del autor con otro derecho, el de la educación", por lo que en la reforma, señalaba tajantemente el Consejo de Estado:

“Debe pues necesariamente señalarse que dicho artículo 32 no será aplicable a los supuestos en los que bien la legislación, bien los titulares, hayan optado por ofrecer a toda la humanidad el acceso abierto de datos y documentos científicos supuestamente sometidos a derechos de propiedad intelectual".

Nada de esto fue aceptado por el Ministerio de Educación, Cultura y Deporte, quien en el texto que llevó finalmente al Consejo de Ministros, que fue aprobado por éste y enviado al Congreso de los Diputados, hizo caso omiso de las recomendaciones del Consejo de Estado, a pesar de que este órgano señalase que en la Unión Europea el open access tiene carácter normativo desde julio de 2012. Se repite de esta manera lo ocurrido con el canon digital: aun cuando la norma estatal sea contraria al derecho de la Unión Europea, las entidades de gestión seguirán cobrando durante el tiempo necesario hasta que se obtenga una resolución del TJUE que anule la legislación del Estado. No podemos olvidar el informe de la Comisión Nacional de la Competencia (CNC, 2009, p. 99) que ya advertía que "no es del todo descartable conforme a la jurisprudencia comunitaria que la Comisión Europea pueda llegar a considerar que España es responsable de una infracción del artículo 86 del Tratado CE (actual art. 106 del Tratado de Funcionamiento de la Unión Europea)".

El corolario de la actual situación es que los hechos nos demuestran que las entidades de gestión, lejos de ser entidades que cumplen su obligación de favorecer la cultura (entendiendo dentro de ella, como no puede ser menos, la ciencia y la tecnología), son lobbies que ocupan en la actualidad el papel que en la llustración fue ocupado por la Iglesia.

Las entidades de gestión, lejos de cumplir su obligación de favorecer la cultura, son lobbies que ocupan en la actualidad el papel que en la llustración fue ocupado por la Iglesia

Nos hallamos en un momento histórico en que la tecnología como entorno (De-la-Cueva-González-Cotera, 2012b) y el open access como contenido (De-la-Cueva-González-Cotera, 2012a) nos permite intentar una Tercera Ilustración y en donde tendremos que reflexionar si no comienza a ser un imperativo ético urgente denunciar y expulsar a quienes son los más piratas. Recordando a Agustín de Hipona:

Mas porque yo ejecuto mis piraterías con un pequeño bajel me llaman ladrón, y a ti, porque las haces con formidables ejércitos, te llaman rey ${ }^{3}$.

\section{Notas}

1. Sobre open access, ver: Ariño-Villarroya, 2009; Lafuente y Alonso, 2011, pp. 193-206; Lafuente, Alonso y Rodríguez; 2013, pp. 16-69.

2. Ello a pesar de que se le solicitó en dos ocasiones al Ministerio de Cultura, que tenía potestad reglamentaria para excluir a quien tuviera conveniente. La primera de ellas, en fecha 19 de noviembre de 2003 por el autor de este artículo y la segunda en fecha 24 de febrero de 2004 por parte de la junta de delegados de la Facultad de Informática de la Universidad Politécnica de Madrid, dado que los alumnos encontraban gravados los soportes en los que debían presentar los trabajos a sus profesores.

http://derecho-internet.org/node/245

3. San Agustín. La ciudad de Dios. Tomo I, Libro cuarto, capítulo IV.

\section{Bibliografía}

Aeval (Agencia Estatal de Evaluación de las Políticas Públicas y la Calidad de los Servicios) (2008). Evaluación del sistema de gestión colectiva de los derechos de propiedad intelectual. http://www.aeval.es/comun/pdf/evaluaciones/E12B.pdf

AIE (Artistas, Intérpretes o Ejecutantes, Sociedad de Gestión) (2010). Memoria AIE 2009.

http://aie.es/component/remository/func-startdown/100 
AIE (Artistas, Intérpretes o Ejecutantes, Sociedad de Gestión) (2011). Memoria AIE 2010.

http://aie.es/component/remository/func-startdown/121

AIE (Artistas, Intérpretes o Ejecutantes, Sociedad de Gestión) (2012). Memoria AIE 2011.

http://aie.es/component/remository/func-startdown/143

AIE (Artistas, Intérpretes o Ejecutantes, Sociedad de Gestión) (2013). Memoria AIE 2012.

http://aie.es/component/remository/func-startdown/39

Aisge (Artistas Intérpretes, Sociedad de Gestión) (2010). Memoria 2009 Aisge.

http://www.aisge.es/media/multimedia/ficheros/204.pdf

Aisge (Artistas Intérpretes, Sociedad de Gestión) (2011). Memoria Aisge 2010.

http://www.aisge.es/media/multimedia/ficheros/275.pdf

Aisge (Artistas Intérpretes, Sociedad de Gestión) (2012). Memoria Aisge 2011.

http://www.aisge.es/media/multimedia/ficheros/319.pdf

Aisge (Artistas Intérpretes, Sociedad de Gestión) (2013). Memoria Aisge 2012.

http://www.aisge.es/media/multimedia/ficheros/333.pdf

Agedi (Asociación de Gestión de Derechos Intelectuales) (2010). Memoria de actividades e informe de gestión 2009. http://www.agedi.es/docs/memoria_e_informe_de_ gestion_agedi_2009.pdf

Agedi (Asociación de Gestión de Derechos Intelectuales) (2011). Memoria de actividades e informe de gestión 2010. http://www.agedi.es/docs/memoria_e_informe_de_ gestion_agedi_2010.pdf

Agedi (Asociación de Gestión de Derechos Intelectuales) (2012). Memoria de actividades e informe de gestión 2011. http://www.agedi.es/docs/memoria_e_informe_de_ gestion_agedi_2011.pdf

Agedi (Asociación de Gestión de Derechos Intelectuales) (2013). Memoria de actividades e informe de gestión 2012. http://www.agedi.es/docs/memoria_e_informe_de_ gestion_agedi_2012.pdf

Ariño-Villarroya, Antonio (2009). El movimiento open. La creación de un dominio público en la era digital. Valencia: Universitat de València. ISBN: 9788437075952

Benkler, Yochai (2006). The wealth of networks. How social production transforms markets and freedom. New Haven \& Londres: Yale University Press. ISBN: 9780300110562

Cedro (Centro Español de Derechos Reprográficos (2010). Memoria 2009.

http://www.cedro.org/docs/memorias-pdf/cedromemoria2009. $p d f$

Cedro (Centro Español de Derechos Reprográficos) (2011). Memoria 2010.

http://www.cedro.org/docs/memorias-pdf/memoria2010.pdf

Cedro (Centro Español de Derechos Reprográficos) (2012). Memoria 2011.

http://www.cedro.org/docs/memorias-pdf/memoria2011.pdf
Cedro (Centro Español de Derechos Reprográficos) (2013). Memoria 2012.

htt $p: / / c e d r o . o r g / d o c s / s o c i o s / m e m o r i a-$ 20124888AEFC5D16C22A353B728D.pdf

CNC (Comisión Nacional de la Competencia) (2009). Informe sobre la gestión colectiva de derechos de propiedad intelectual. http://goo.gl/gM3le2

Consejo de Estado (2013). Dictamen 1064/2013 (Educación, Cultura y Deporte) sobre Anteproyecto de ley de modificación del texto refundido de la Ley de propiedad intelectual, aprobado por Real decreto legislativo 1/1996, de 12 de abril, y de la Ley 1/2000, de 7 de enero, de Enjuiciamiento civil. https://www.boe.es/buscar/doc.php?id=CE-D-2013-1064

CRUE (Conferencia de Rectores de las Universidades Españolas) (2013). Alegaciones al Anteproyecto de ley de modificación del texto refundido de la Ley de propiedad intelectual, aprobado por Real decreto legislativo 1/1996, de 12 de abril, y de la Ley $1 / 2000$, de 7 de enero, de Enjuiciamiento civil. http://goo.gl/3JGACG

De-la-Cueva-González-Cotera, Javier (2012a). “Innovación y conocimiento libre: cuestiones morales y políticas". Isegoría. Revista de filosofía moral y política, n. 48, pp. 51-74.

http://isegoria.revistas.csic.es/index.php/isegoria/article/ view/810/809

http://dx.doi.org/10.3989/isegoria.2013.048.03

De-la-Cueva-González-Cotera, Javier (2012b). “Internet como entorno de la opinión pública: envolviendo los derechos fundamentales en derechos ordinarios". Revista internacional de pensamiento político, n. 7, pp. 93-115. http://www.pensamientopolitico.org/Descargas/ RIPP07093115.pdf

Dama (Derechos de Autor de Medios Audiovisuales) (2011). Memoria e informe de gestión 2010.

http://damautor.es/pdf/memoria_2010.pdf

Dama (Derechos de Autor de Medios Audiovisuales) (2012). Memoria e informe de gestión 2011.

http://damautor.es/pdf/MEMORIA\%20DAMA\%202011.pdf

Dama (Derechos de Autor de Medios Audiovisuales) (2013). Memoria e informe de gestión 2012.

http://damautor.es/pdf/MEMORIA2012.pdf

España (1996). "Real decreto legislativo 1/1996, de 12 de abril, por el que se aprueba el texto refundido de la Ley de propiedad intelectual, regularizando, aclarando y armonizando las disposiciones legales vigentes sobre la materia". Boletín oficial del estado, 22 de abril, n. 97, pp. 14.36914.396.

https://www.boe.es/diario_boe/txt.php?id=BOE-A-1996-8930

España (2011). "Real decreto-ley 20/2011, de 30 de diciembre, de medidas urgentes en materia presupuestaria, tributaria y financiera para la corrección del déficit público". Boletín oficial del estado, 30 de diciembre, n. 315, pp. 146.574-146.648.

https://www.boe.es/diario_boe/txt.php?id=BOE-A-2011-20638

España (2012). "Real decreto 1657/2012, de 7 de diciembre, por el que se regula el procedimiento de pago de la com- 
pensación equitativa por copia privada con cargo a los Presupuestos generales del Estado". Boletín oficial del estado, 7 de diciembre, n. 295, pp. 84.141-84.148.

http://www.boe.es/diario_boe/txt.php?id=BOE-A-2012-14904

España (2013). “Orden ECD/2128/2013, de 14 de noviembre, por la que se determina la cuantía de la compensación equitativa por copia privada correspondiente al ejercicio 2012 con cargo a los Presupuestos generales del Estado y su distribución entre las tres modalidades de reproducción referidas legalmente". Boletín oficial del estado, 16 de noviembre, n. 275, pp. 91797-91799.

https://www.boe.es/buscar/doc.php?id=BOE-A-2013-12021

Ferrajoli, Luigi (2010). Derechos y garantías. La ley del más débil. Madrid: Editorial Trotta. ISBN: 9788481642858

Gutiérrez, Bernardo (2012). "Entrevista a Antonio Lafuente: Los hackers son los científicos de la nueva Ilustración". Código abierto, 23 enero.

http://goo.gl/cFB9XM

Lafuente, Antonio; Alonso, Andoni (2011). Ciencia expandida, naturaleza común y saber profano. Bernal (Argentina): Universidad Nacional de Quilmes. ISBN: 9789875582125

Lafuente, Antonio; Alonso, Andoni; Rodríguez, Joaquín (2013). iTodos sabios! Ciencia ciudadana y conocimiento expandido. Madrid: Cátedra, 2013. ISBN: 9788437630717

Sgae (Sociedad General de Autores y Editores) (2010). Informe de gestión y responsabilidad social corporativa 2009 de la SGAE. http://www.sgae.es/recursos/informes/IGRSC_SGAE_2009.pdf

Sgae (Sociedad General de Autores y Editores) (2011). Informe de gestión y responsabilidad social corporativa 2010 de la SGAE. http://www.sgae.es/recursos/informes/memoria_2010/ IGRSC_SGAE_2010op.pdf

Sgae (Sociedad General de Autores y Editores) (2012). Infor- me de gestión 2011.

http://www.sgae.es/recursos/Memoria_2011/Informe_de_ gestion_SGAE_2011.pdf

Sgae (Sociedad General de Autores y Editores) (2013). Informe de gestión 2012.

http://www.sgae.es/recursos/Memoria_2012/gestion.html

Tribunal de Justicia de la Unión Europea (2010). Caso Padawan (C-467/08). Sentencia de 21 de octubre de 2010.

Unión Europea (2001). “Directiva 2001/29/CE del Parlamento Europeo y del Consejo, de 22 de mayo de 2001, relativa a la armonización de determinados aspectos de los derechos de autor y derechos afines a los derechos de autor en la sociedad de la información". Diario oficial de la Unión Europea L 167, 22 de junio, pp. 10-19.

http://eur-lex.europa.eu/LexUriServ/LexUriServ.do?uri=OJ:L :2001:167:0010:0019:ES:PDF

Vegap (Visual, Entidad de Gestión de Artistas Plásticos) (2010). Memoria 2009.

http://www.vegap.es/Info/Documentos/MEMORIASVEGAP/memoria-vegap-2009.pdf

Vegap (Visual, Entidad de Gestión de Artistas Plásticos) (2011). Memoria 2010.

http://www.vegap.es/Info/Documentos/MEMORIASVEGAP/memoria-vegap-2010.pdf

Vegap (Visual, Entidad de Gestión de Artistas Plásticos) (2012). Memoria 2011.

http://www.vegap.es/Info/Documentos/MEMORIASVEGAP/memoria-vegap-2011.pdf

Vegap (Visual, Entidad de Gestión de Artistas Plásticos) (2013). Memoria 2012.

http://www.vegap.es/Info/Documentos/MEMORIASVEGAP/memoria-vegap-2012.pdf

\section{Próximos temas centrales}

$\begin{array}{ll}\text { Mayo 2014 } & \text { Visualización de información } \\ \text { Julio 2014 } & \text { Altmétricas } \\ \text { Septiembre 2014 } & \text { Humanidades digitales } \\ \text { Noviembre 2014 } & \text { Big data y analítica web } \\ \text { Enero 2015 } & \text { Marketing de productos de información } \\ \text { Marzo 2015 } & \text { Web semántica } \\ \text { Mayo 2015 } & \text { Privacidad y seguridad de información }\end{array}$

Los interesados por favor consulten detenidamente las Normas para autores:

http://www.elprofesionaldelainformacion.com/autores.html

y luego envíen sus artículos a través del gestor de manuscritos OJS de la plataforma del Recyt: http://recyt.fecyt.es/index.php/EPI/index 